\title{
Management of sagittal synostosis in the Synostosis Research Group: baseline data and early outcomes
}

\author{
Cordell M. Baker, MD, ${ }^{1}$ Vijay M. Ravindra, MD, MSPH, ${ }^{1-3}$ Barbu Gociman, MD, PhD, ${ }^{4}$ \\ Faizi A. Siddiqi, MD, ${ }^{4}$ Jesse A. Goldstein, MD, ${ }^{5}$ Matthew D. Smyth, MD, ${ }^{6}$ Amy Lee, MD, \\ Richard C. E. Anderson, MD, ${ }^{8}$ Kamlesh B. Patel, MD, MSc, ${ }^{9}$ Craig Birgfeld, MD, ${ }^{10}$ \\ Ian F. Pollack, MD, ${ }^{10}$ Thomas Imahiyerobo, MD, ${ }^{11}$ and John R. W. Kestle, MD, ${ }^{1}$ \\ for the Synostosis Research Group
}

Divisions of ${ }^{1}$ Pediatric Neurosurgery, Primary Children's Hospital, and ${ }^{4}$ Plastic and Reconstructive Surgery, University of Utah, Salt Lake City, Utah; ' $D$ Division of Neurosurgery, University of California, San Diego, California; ${ }^{2}$ Department of Neurosurgery, Naval Medical Center San Diego, California; Departments of ${ }^{5}$ Plastic Surgery and ${ }^{10}$ Pediatric Neurosurgery, UPMC Children's Hospital of Pittsburgh, Pennsylvania; ${ }^{6}$ Department of Neurosurgery and ${ }^{9}$ Division of Plastic and Reconstructive Surgery, Department of Surgery, St. Louis Children's Hospital, Washington University School of Medicine in St. Louis, Missouri; ${ }^{7}$ Department of Neurosurgery, Seattle Children's Hospital, University of Washington, Seattle, Washington; ${ }^{8}$ Department of Neurosurgery, Columbia University, Morgan Stanley Children's Hospital, New York; and ${ }^{11}$ Division of Plastic Surgery, Columbia University Medical Center, NewYork-Presbyterian Hospital, New York, New York

OBJECTIVE Sagittal synostosis is the most common form of isolated craniosynostosis. Although some centers have reported extensive experience with this condition, most reports have focused on a single center. In 2017, the Synostosis Research Group (SynRG), a multicenter collaborative network, was formed to study craniosynostosis. Here, the authors report their early experience with treating sagittal synostosis in the network. The goals were to describe practice patterns, identify variations, and generate hypotheses for future research.

METHODS All patients with a clinical diagnosis of isolated sagittal synostosis who presented to a SynRG center between March 1, 2017, and October 31, 2019, were included. Follow-up information through October 31, 2020, was included. Data extracted from the prospectively maintained SynRG registry included baseline parameters, surgical adjuncts and techniques, complications prior to discharge, and indications for reoperation. Data analysis was descriptive, using frequencies for categorical variables and means and medians for continuous variables.

RESULTS Two hundred five patients had treatment for sagittal synostosis at 5 different sites. One hundred twenty-six patients were treated with strip craniectomy and 79 patients with total cranial vault remodeling. The most common strip craniectomy was wide craniectomy with parietal wedge osteotomies (44\%), and the most common cranial vault remodeling procedure was total vault remodeling without forehead remodeling (63\%). Preoperative mean cephalic indices (Cls) were similar between treatment groups: 0.69 for strip craniectomy and 0.68 for cranial vault remodeling. Thirteen percent of patients had other health problems. In the cranial vault cohort, $81 \%$ of patients who received tranexamic acid required a transfusion compared with $94 \%$ of patients who did not receive tranexamic acid. The rates of complication were low in all treatment groups. Five patients $(2 \%)$ had an unintended reoperation. The mean change in $\mathrm{Cl}$ was 0.09 for strip craniectomy and 0.06 for cranial vault remodeling; wide craniectomy resulted in a greater change in $\mathrm{Cl}$ in the strip craniectomy group.

CONCLUSIONS The baseline severity of scaphocephaly was similar across procedures and sites. Treatment methods varied, but cranial vault remodeling and strip craniectomy both resulted in satisfactory postoperative Cls. Use of tranexamic acid may reduce the need for transfusion in cranial vault cases. The wide craniectomy technique for strip craniectomy seemed to be associated with change in $\mathrm{Cl}$. Both findings seem amenable to testing in a randomized controlled trial.

https://thejns.org/doi/abs/10.3171/2021.1.FOCUS201029

KEYWORDS sagittal synostosis; craniosynostosis; strip craniectomy; cranial vault reconstruction; Synostosis Research Group

ABBREVIATIONS CI = cephalic index; SynRG = Synostosis Research Group; TXA = tranexamic acid .

SUBMITTED December 4, 2020. ACCEPTED January 5, 2021.

INCLUDE WHEN CITING DOI: 10.3171/2021.1.FOCUS201029. 
$\mathrm{W}$ ITH an estimated incidence of 1 in 2000 persons, sagittal synostosis is the most common form of isolated craniosynostosis, accounting for roughly half of all synostosis cases. ${ }^{1}$ The first description of surgical treatment of sagittal synostosis-involving strip craniectomy - was in $1892 .^{2}$ Although current surgical treatments involve a similar approach with removal of the synostotic suture, a variety of opinions on optimal surgical techniques and adjuvants have been developed.,2,3 Variations in treatment include strip craniectomy with or without helmet therapy, endoscope-assisted surgery, cranial vault remodeling with or without the modified pi technique, forehead reshaping, use of preoperative and postoperative imaging, and spring- or distractor-assisted craniectomy. Despite the disparity in treatments, there remains a paucity of information regarding the best practices for sagittal synostosis treatment.

Although multiple centers have reported extensive experience with this condition, evidence to guide the development of best practices is limited because the literature comprises mostly single-center studies reporting their preferred treatment paradigm. ${ }^{4-6}$ In 2017, the Synostosis Research Group (SynRG) was established as a multicenter collaborative network to study craniosynostosis. The SynRG consists of 5 sites, with at least one plastic surgeon and one neurosurgeon at each site, that prospectively collect data on children diagnosed with craniosynostosis. In a previous survey, we asked surgeons to describe their usual practice patterns in the treatment of sagittal synostosis. ${ }^{3}$ Here, we present prospective observational data using standardized data fields and a centralized database to describe our early experience with treating sagittal synostosis in the network. The goal of this study was to describe practice patterns, identify variations, and generate hypotheses for future research.

\section{Methods}

Local institutional review board approval was received at each site and allowed data collection with a waiver of patient consent. All patients with an initial clinical diagnosis of isolated sagittal synostosis who presented to a SynRG center between March 1, 2017, and October 31, 2019, were included. Patient information at the most recent followup or first reoperation through October 31, 2020, was included. Data extracted from the SynRG registry included baseline parameters, surgical adjuncts and techniques, complications before discharge, and indications for reoperation. Data analysis was descriptive, using numbers and frequencies for categorical variables, medians and means for continuous variables, and means with $95 \%$ confidence intervals for cephalic index (CI) data. All analyses were performed using IBM SPSS (version 24.0, IBM Corp.).

\section{Results}

\section{Preoperative Assessment}

There were 205 children (53 females and 152 males; mean age at operation 7.8 months) who had the clinical features of sagittal synostosis diagnosed during the study dates (Table 1). Diagnosis was confirmed with CT scanning in 183 patients (89\%). More patients were treated with strip craniectomy (126 patients) - with either spring placement (26 patients) or postoperative helmet therapy (100 patients) - than cranial vault remodeling (79 patients). The mean baseline CIs were similar across groups: 0.69 for patients who underwent strip craniectomy and 0.68 for patients who underwent cranial vault remodeling. The mean baseline CIs were also similar across treatment sites, ranging from 0.67 to 0.69 , indicating similar disease severity across sites. Older children were more likely to

TABLE 1. Characteristics of patients treated with strip craniectomy or cranial vault surgery

\begin{tabular}{lccc}
\hline \multicolumn{1}{c}{ Characteristic } & Strip Craniectomy & Cranial Vault Surgery & Total \\
\hline Patients & $126(61)$ & $79(39)$ & $205(100)$ \\
\hline Mean age at op \pm SD, mos & $3.4 \pm 1.0$ & $14.7 \pm 14.7$ & $7.8 \pm 10.7$ \\
\hline Patient age group, mos & & & \\
\hline$<3$ & $51(40)$ & 0 & $51(25)$ \\
\hline $3-6$ & $74(59)$ & $18(23)$ & $92(45)$ \\
\hline$>6$ & $1(1)$ & $61(77)$ & $62(30)$ \\
\hline Female sex & $35(28)$ & $18(23)$ & $53(26)$ \\
\hline Preop imaging completed & $105(83)$ & $78(99)$ & $183(89)$ \\
\hline Additional fused suture on imaging & $3(2)$ & $3(4)$ & $6(3)$ \\
\hline Mean baseline Cl & 0.69 & 0.68 & 0.69 \\
\hline Mean baseline occipitofrontal circumference, percentile & 82.3 & 84.7 & 83.2 \\
\hline Preop visual evoked potentials & $9(7)$ & $2(3)$ & $11(6)$ \\
\hline Other health problem* & $14(11)$ & $12(15)$ & $26(13)$ \\
\hline Family history of synostosis & $10(8)$ & $6(8)$ & $16(8)$ \\
\hline Parental developmental concerns & $1(1)$ & $6(8)$ & $7(3)$ \\
\hline $\begin{array}{l}\text { Values are shown as } n \text { (\%) unless indicated otherwise. } \\
\text { * Included neurological (3.9\%), cardiovascular (3.4\%), gastrointestinal (3.9\%), genetic (3.9\%), respiratory, renal and urological, hematological or } \\
\text { immunological, and metabolic conditions. }\end{array}$ & &
\end{tabular}


TABLE 2. Family history of synostosis

\begin{tabular}{cccc}
\hline & \multicolumn{3}{c}{ No. of Patients (\%) } \\
\cline { 2 - 4 } Suture Type & Parent & Sibling & Other \\
\hline Sagittal & $3(2)$ & $2(1)$ & $9(4)$ \\
\hline Other & $2(1)$ & 0 & $3(2)$ \\
\hline
\end{tabular}

be treated with cranial vault remodeling (mean age 14.7 months) than strip craniectomy (mean age 3.4 months).

At the time of their baseline assessments, 26 children $(13 \%)$ had one or more other known health problems, including neurological (4\%), gastrointestinal (4\%), genetic $(4 \%)$, or cardiovascular (3\%) conditions. The rates of other health problems were similar in the 2 treatment groups: $11 \%$ in patients who underwent strip craniectomy versus $15 \%$ in patients who underwent cranial vault remodeling. Parents expressed developmental concerns for 7 children $(3 \%), 6$ of whom were treated with cranial vault remodeling. Overall, 16 patients $(8 \%)$ had a family history of synostosis, with the majority of affected family members having sagittal synostosis (Table 2).

In $6(3 \%)$ of the 183 patients who underwent preoperative imaging, an additional fused suture was found. Base- line visual evoked potentials were assessed in 12 patients (6\%), and no children underwent formal neuropsychological testing before surgery.

\section{Surgical Technique and Perioperative Management}

Surgical technique varied by site. One site described only 3 procedures in the database, and they were all strip craniectomies followed by helmet therapy. At the other 4 sites, $7.7 \%$ of cases were treated with strip craniectomy at 1 site compared with $62 \%, 80 \%$, and $81 \%$ at the other 3 sites.

Surgical technique characteristics across the cohort are shown in Table 3 . The strip craniectomy techniques $(\mathrm{n}=126)$ included 1$)$ wide craniectomy with 2 wedge osteotomies in the parietal bone followed by helmet therapy (55 children [44\%]), 2) narrow craniectomy with no parietal osteotomies followed by helmet therapy (46 children [37\%]), and 3) narrow craniectomy and insertion of springs without helmet therapy (25 children [20\%]). An endoscope was used in $81 \%$ of strip craniectomy cases. The cranial vault remodeling procedures $(n=79)$ comprised pi procedures (11 children [14\%]), reverse pi procedures (3 children [4\%]), posterior two-thirds cranial vault remodeling without forehead remodeling (50 children [63\%]), and total cranial vault remodeling including the forehead (15

TABLE 3. Intraoperative characteristics of strip craniectomy and cranial vault surgery

\begin{tabular}{|c|c|c|}
\hline \multirow[b]{2}{*}{ Characteristic } & \multicolumn{2}{|c|}{ No. of Patients (\%) } \\
\hline & Strip Craniectomy & Cranial Vault Surgery \\
\hline \multicolumn{3}{|l|}{ Procedure type } \\
\hline Wide craniectomy w/ parietal wedge osteotomies & $55(44)$ & \\
\hline Narrow craniectomy w/o parietal osteotomy & $46(37)$ & \\
\hline Narrow craniectomy w/ springs & $25(20)$ & \\
\hline Endoscope used & $102(81)$ & \\
\hline For only epidural dissection & $65(64)$ & \\
\hline For epidural dissection \& bone cautery & $37(36)$ & \\
\hline \multicolumn{3}{|l|}{ Pi procedure used } \\
\hline Modified & & $11(14)$ \\
\hline Reverse & & $3(4)$ \\
\hline \multicolumn{3}{|l|}{ Cranial vault reconstruction } \\
\hline w/o forehead remodeling & & $50(63)$ \\
\hline w/ forehead remodeling & & $15(19)$ \\
\hline \multicolumn{3}{|l|}{ Patient position } \\
\hline Sphinx & $94(75)$ & $32(41)$ \\
\hline Prone & $25(20)$ & $40(51)$ \\
\hline Supine & $5(4)$ & $6(8)$ \\
\hline Antibiotics prior to incision & $122(97)$ & $79(100)$ \\
\hline \multicolumn{3}{|l|}{ Line inserted } \\
\hline Arterial & $43(34)$ & $69(88)$ \\
\hline Central & 0 & $3(4)$ \\
\hline Foley catheter & $11(9)$ & $64(81)$ \\
\hline Intraop steroids & $72(60)$ & $70(91)$ \\
\hline TXA & $7(6)$ & $62(78)$ \\
\hline Precordial Doppler & $6(5)$ & $35(44)$ \\
\hline Cell salvage & $1(1)$ & $5(6)$ \\
\hline
\end{tabular}


TABLE 4. Postoperative management following strip craniectomy and cranial vault surgery

\begin{tabular}{lcc}
\hline Postop Technique & Strip Craniectomy & Cranial Vault Surgery \\
\hline Subgaleal drain & 0 & $74(94)$ \\
\hline Admitted to ICU & $13(10)$ & $79(100)$ \\
\hline $\begin{array}{l}\text { CT before discharge } \\
\text { from hospital }\end{array}$ & $8(8)$ & $43(59)$ \\
\hline $\begin{array}{l}\text { Length of stay, days } \\
\text { Median }\end{array}$ & 1 & 3 \\
\hline Mean & 1.8 & 3.2 \\
\hline Postop helmet use & $100(79)$ & NA \\
\hline
\end{tabular}

$\mathrm{NA}=$ not applicable.

Values are shown as $n(\%)$ unless indicated otherwise.

children [19\%]). The sphinx position (75\%) was preferred for strip craniectomy techniques, and the prone position (51\%) was preferred for cranial vault procedures.

Perioperative interventions differed depending on the type of surgery performed (Table 3). Adjuncts were used more often in cranial vault remodeling than strip craniectomy, although the difference was negligible in some cases. The placement of an arterial line was common $(55 \%$ overall), but more so during cranial vault procedures (88\%) than strip craniectomies (34\%). A Foley catheter was used in $37 \%$ of cases (9\% of strip craniectomies and $81 \%$ of cranial vault remodeling procedures). Tranexamic acid (TXA) and precordial Doppler were used in the majority of cranial vault remodeling procedures. Central lines and cell salvage were rarely used in either surgery. These practice patterns did not vary substantially among sites, except use of TXA. It was almost always used during cranial vault remodeling at 3 sites but only rarely at the other 2 institutions. The intraoperative transfusion rate was $81 \%$ when TXA was used compared with $94 \%$ when it was not used.

Postoperative management data are shown in Table 4. Postoperative drains were not used in patients who under- went strip craniectomy but were almost always used in patients who underwent cranial vault remodeling (94\%). Postoperatively, all patients who underwent cranial vault remodeling were admitted to the pediatric intensive care unit, whereas only $10 \%$ of patients who underwent strip craniectomy required intensive care. The length of stay also varied between groups: the median length of stay was 1 day for patients who underwent strip craniectomy versus 3 days for patients who underwent cranial vault remodeling. Over half $(59 \%)$ of patients who underwent cranial vault remodeling underwent CT scanning before discharge, whereas only $8 \%$ of patients who underwent strip craniectomy underwent imaging before discharge. All patients in the strip craniectomy group who underwent surgery without springs (100 patients) were treated with a postoperative helmet.

\section{Unintended Events and Outcomes}

Intraoperative complications were rare (Table 5). Dural opening occurred during 1 strip surgery $(1 \%)$ and 3 cranial vault remodeling procedures (4\%). Bleeding from the sagittal sinus was seen during 2 strip craniectomies $(2 \%)$ and 1 vault surgery (1\%). There were no air emboli or brain injuries. Intraoperative transfusions were more common in the cranial vault cohort (83\%) than in the strip craniectomy cohort (7\%). Additional complications were also rare before hospital discharge. In the cranial vault cohort, there were 2 patients with subdural hematoma and $1 \mathrm{pa}-$ tient with epidural hematoma, but none required intervention. Two patients who underwent strip craniectomy and 1 patient who underwent cranial vault remodeling required a blood transfusion before discharge, neither of whom had received an intraoperative transfusion. Repeat surgery occurred in 32 children. Repeat surgery was expected for device removal in the 25 patients who underwent springassisted craniectomy and 1 patient who underwent distractor-assisted craniectomy. There were 5 unplanned reoperations for spring repositioning (1 patient), infection after strip surgery (1 patient), delayed hematoma after strip sur-

TABLE 5. Unintended perioperative events and reoperations

\begin{tabular}{|c|c|c|c|}
\hline \multirow[b]{2}{*}{ Event Type } & \multicolumn{3}{|c|}{ No. of Patients (\%) } \\
\hline & Strip Craniectomy & Cranial Vault Surgery & Total \\
\hline Durotomy & $1(1)$ & $3(4)$ & $4(2)$ \\
\hline Transfusion & $9(7)$ & $66(83)$ & $75(37)$ \\
\hline Sinus bleeding & $2(2)$ & $1(1)$ & $3(1)$ \\
\hline Air embolus & 0 & 0 & 0 \\
\hline Brain injury & 0 & 0 & 0 \\
\hline Postop subdural blood on imaging & 0 & $1(1)$ & $1(1)$ \\
\hline Postop epidural blood on imaging & 0 & $2(3)$ & $2(1)$ \\
\hline Postop bone defect $>2 \mathrm{~cm}$ at follow-up $>6$ mos & $12(10)$ & $4(5)$ & $16(8)$ \\
\hline \multicolumn{4}{|l|}{ Unplanned reop } \\
\hline Additional suture synostosis & $2(2)$ & 0 & $2(1)$ \\
\hline Infection & $1(1)$ & 0 & $1(0.5)$ \\
\hline Hematoma & $1(1)$ & 0 & $1(0.5)$ \\
\hline Spring repositioning & $1(1)$ & 0 & $1(0.5)$ \\
\hline
\end{tabular}


TABLE 6. Cl outcomes

\begin{tabular}{lcrc}
\hline \multicolumn{1}{c}{ Cl } & Strip Craniectomy & Cranial Vault Surgery & Total \\
\hline Final & $0.79(0.78-0.80)$ & $0.75(0.73-0.77)$ & 0.77 \\
\hline Change & $0.09(0.08-0.10)$ & $0.06(0.03-0.08)$ & 0.09 \\
\hline Change w/ wide craniectomy* & $0.11(0.10-0.13)$ & & \\
\hline Change w/ narrow craniectomy $\dagger$ & $0.06(0.05-0.08)$ & & \\
\hline Values are shown as the mean (95\% confidence interval). & & \\
* Defined as $\geq 4 \mathrm{~cm}$ with parietal wedge osteotomies. \\
$\dagger$ Defined as $\leq 2.5 \mathrm{~cm}$ without parietal osteotomy.
\end{tabular}

gery (1 patient), and delayed fusion of an additional suture after strip surgery (2 patients). There were no reoperations for cranial defect after any strip craniectomy or cranial vault surgery.

Change in CI, from baseline to last follow-up, appeared to vary with surgical technique (Table 6). Wide craniectomy $(\geq 4.0 \mathrm{~cm})$ with parietal wedge osteotomies was associated with a change in $\mathrm{CI}$ of 0.11 , and narrow craniectomy $(\leq 2.5 \mathrm{~cm})$ with no parietal osteotomies was associated with a change of 0.06. CI data were unavailable for the 25 spring-assisted procedures. After cranial vault surgery, the mean change in CI was 0.06 .

Almost all parents in both groups scored the head shape of the patient on follow-up as "much better," with $94 \%$ of parents in the cranial vault cohort and $86 \%$ in the strip craniectomy cohort describing the outcome as such. Three percent of the parents in the cranial vault cohort and $9 \%$ of those in the strip craniectomy cohort described the head shape as "a little better," and only 5\% and 3\%, respectively, thought the results were the "same." None of the parents reported a worse shape.

\section{Discussion}

The purpose of this study was to identify areas of commonality and practice variations among treatment sites, with the hope that these data will prompt further research. Not surprisingly, we found variations in many aspects of sagittal synostosis management, including surgical timing and technique and use of intraoperative TXA. We had no required protocols for perioperative patient management, and although we wanted to identify areas of variation, such variation potentially confounded our assessment of outcomes. Equally interesting was the similarity of disease severity, with preoperative CIs being remarkably consistent in patients who underwent cranial vault remodeling and strip craniectomy across sites. Similar baseline CIs are advantageous when comparing changes in CI across treatment modalities and centers.

Sagittal synostosis is commonly thought of as an isolated health event that is not associated with other medical conditions. We recorded the presence of other complex chronic conditions in patients treated for sagittal synostosis. We found that approximately 13\% of children (or 1 in 8) with sagittal synostosis had at least one other health issue; the most common of these were gastrointestinal, genetic, and other neurological conditions. The potential for other problems, particularly cardiac and neurological, may impact surgery and outcomes and should be consid- ered when planning the procedure. An additional fused suture on CT is another preoperative observation that may affect clinical treatment. In our study, 6 patients (3\%) were found to have an additional fused suture; this is similar to another study in which 7 (5\%) of 139 patients were found to have multiple-suture synostosis on preoperative evaluation for sagittal synostosis.?

We have identified multiple areas of interest that could be studied more formally in the future: 1) our data suggest that surgical technique and magnitude of scaphocephaly correction may be related, and 2) use of TXA to minimize blood loss and transfusion may be beneficial in cranial vault remodeling surgery.

Children treated with wide craniectomy, defined as craniectomy $\geq 4 \mathrm{~cm}$, and parietal wedge osteotomies had a mean improvement in CI of 0.11 , whereas those treated with narrow craniectomy without parietal osteotomies improved by 0.06 . This contrasts with the results of Dlouhy et al. ${ }^{8}$ who found no difference between endoscope-assisted wide craniectomy with barrel-stave osteotomies and narrow craniectomy in a prospective study of 28 patients. In that study, the authors defined craniectomy widths as 4-6 $\mathrm{cm}$ and $2 \mathrm{~cm}$, respectively. ${ }^{8}$ Another single-center study that utilized narrow craniectomy with adjuvant helmet therapy showed similar results to those of our study, with a mean change in CI of 0.07 and a mean final CI of 0.76 in 61 patients. ${ }^{9}$ In a comparison of cranial vault procedures with strip craniectomy, a large systematic review of 27 studies found that both treatment modalities had varying degrees of improvement in CI, but there was not enough evidence to suggest either treatment group had greater improvement than the other..$^{10}$ The lower cost associated with strip surgery has been documented. ${ }^{11}$ The choice of operative technique for correction of scaphocephaly remains controversial and should be studied further.

Multiple studies have been published on the utility of TXA to reduce blood loss in craniosynostosis surgery. ${ }^{12-14}$ These studies are retrospective but show favorable results, with decreased rates of intraoperative blood loss, red blood cell transfusion volume, and plasma and platelet transfusion. ${ }^{12,14}$ Consistent with these results, our study revealed that $81 \%$ of children required transfusion when TXA was used in cranial vault remodeling compared with $94 \%$ when TXA was not used. These other studies did not report significant complications associated with TXA use, but the assessment levels of these studies remain unclear. Certainly, in the nonsynostosis literature, complications such as stroke and seizure have been reported with TXA use..$^{15-17}$ 
In our analysis, we intentionally did not use statistical tests. We did not believe they were justified because this is an initial observational study without a priori hypotheses or sample size. We sought to simply describe our early experience and obtain event rates that will allow the development of testable hypotheses for further work. We have also identified areas for improvement of our network. Consistent use of the same outcome measure is essential. We thought that $\mathrm{CI}$ would be a simple universal outcome, but it was not part of the practice at 1 site, and another site used it inconsistently. This limited our sample size for assessing change in CI. In addition, although its quantification is simple, CI does not reflect frontal bossing or pterional narrowing very well. More complicated measurements could be considered but often require postoperative $\mathrm{CT},{ }^{18}$ which was not done consistently at our centers and results in additional radiation exposure. Finally, 1 site had personnel changes soon after joining the network, resulting in a limited number of included patients. We believe that these issues can be corrected and that the advantages of multicenter collaboration, using common data fields, definitions, and common study questions, will provide useful data for the management of these children.

\section{Acknowledgments}

We thank the SynRG site research coordinators Diego Morales at St. Louis Children's Hospital, Kimberly Diamond at UPMC Children's Hospital of Pittsburgh, and Gloria Bowen at Seattle Children's Hospital, and the SynRG network managers Nichol Nunn and Jason Clawson at the University of Utah. We also thank Kristin Kraus for editorial assistance.

\section{References}

1. Massimi L, Caldarelli M, Tamburrini G, et al. Isolated sagittal craniosynostosis: definition, classification, and surgical indications. Childs Nerv Syst. 2012;28(9):1311-1317.

2. Doumit GD, Papay FA, Moores N, Zins JE. Management of sagittal synostosis: a solution to equipoise. J Craniofac Surg. 2014;25(4):1260-1265.

3. Kestle JRW, Lee A, Anderson RCE, et al. Variation in the management of isolated craniosynostosis: a survey of the Synostosis Research Group. J Neurosurg Pediatr. 2018;22(6): 627-631.

4. Chan JW, Stewart CL, Stalder MW, et al. Endoscope-assisted versus open repair of craniosynostosis: a comparison of perioperative cost and risk. J Craniofac Surg. 2013;24(1): 170-174.

5. Panchal J, Marsh JL, Park TS, et al. Sagittal craniosynostosis outcome assessment for two methods and timings of intervention. Plast Reconstr Surg. 1999;103(6):1574-1584.

6. Shah MN, Kane AA, Petersen JD, et al. Endoscopically assisted versus open repair of sagittal craniosynostosis: the St. Louis Children's Hospital experience. J Neurosurg Pediatr. 2011;8(2):165-170.

7. Jimenez DF, Barone CM, McGee ME, et al. Endoscopyassisted wide-vertex craniectomy, barrel stave osteotomies, and postoperative helmet molding therapy in the management of sagittal suture craniosynostosis. J Neurosurg. 2004;100(5 Suppl Pediatrics):407-417.

8. Dlouhy BJ, Nguyen DC, Patel KB, et al. Endoscope-assisted management of sagittal synostosis: wide vertex suturectomy and barrel stave osteotomies versus narrow vertex suturectomy. J Neurosurg Pediatr. 2016;25(6):674-678.
9. Berry-Candelario J, Ridgway EB, Grondin RT, et al. Endoscope-assisted strip craniectomy and postoperative helmet therapy for treatment of craniosynostosis. Neurosurg Focus. 2011;31(2):E5.

10. Thwin M, Schultz TJ, Anderson PJ. Morphological, functional and neurological outcomes of craniectomy versus cranial vault remodeling for isolated nonsyndromic synostosis of the sagittal suture: a systematic review. JBI Database Syst Rev Implement Reports. 2015;13(9):309-368.

11. Garber ST, Karsy M, Kestle JRW, et al. Comparing outcomes and cost of 3 surgical treatments for sagittal synostosis: a retrospective study including procedure-related cost analysis. Neurosurgery. 2017;81(4):680-687.

12. Martin DT, Gries H, Esmonde N, et al. Implementation of a tranexamic acid protocol to reduce blood loss during cranial vault remodeling for craniosynostosis. J Craniofac Surg. 2016;27(6):1527-1531.

13. Kurnik NM, Pflibsen LR, Bristol RE, Singh DJ. Tranexamic acid reduces blood loss in craniosynostosis surgery. J Craniofac Surg. 2017;28(5):1325-1329.

14. Crantford JC, Wood BC, Claiborne JR, et al. Evaluating the safety and efficacy of tranexamic acid administration in pediatric cranial vault reconstruction. J Craniofac Surg. 2015; 26(1):104-107.

15. Maeda T, Sasabuchi Y, Matsui H, et al. Safety of tranexamic acid in pediatric cardiac surgery: a nationwide database study. J Cardiothorac Vasc Anesth. 2017;31(2):549-553.

16. Zhou ZF, Zhang FJ, Huo YF, et al. Intraoperative tranexamic acid is associated with postoperative stroke in patients undergoing cardiac surgery. PLoS One. 2017;12(5):e0177011.

17. Martin K, Wiesner G, Breuer T, et al. The risks of aprotinin and tranexamic acid in cardiac surgery: a one-year followup of 1188 consecutive patients. Anesth Analg. 2008;107(6): 1783-1790.

18. Liaw WXZ, Parr WCH, Peltz TS, et al. Quantification of head shape and cranioplasty outcomes: six-compartment volume method applied to sagittal synostosis. Plast Reconstr Surg Glob Open. 2019;7(4):e2171.

\section{Disclaimer}

The views expressed herein are those of the author(s) and do not necessarily reflect the official policy or position of the Department of the Navy, Department of Defense, or U.S. Government.

\section{Disclosures}

Dr. Patel: consultant for Stryker CMF.

\section{Author Contributions}

Conception and design: Kestle. Acquisition of data: all authors. Analysis and interpretation of data: Kestle, Baker, Ravindra. Drafting the article: Kestle, Baker. Critically revising the article: all authors. Reviewed submitted version of manuscript: all authors. Approved the final version of the manuscript on behalf of all authors: Kestle. Administrative/technical/material support: Kestle, Gociman, Siddiqi, Goldstein, Smyth, Lee, Anderson, Patel, Birgfeld, Pollack, Imahiyerobo.

\section{Correspondence}

John R. W. Kestle: University of Utah, Salt Lake City, UT. neuropub@hsc.utah.edu. 